\title{
Dose reduction for CT coronary calcium scoring with a calcium-aware image reconstruction technique: a phantom study
}

\author{
Ronald Booij ${ }^{1}$ (D) Niels R. van der Werf ${ }^{1,2} \cdot$ Ricardo P. J. Budde $^{1} \cdot$ Daniel Bos ${ }^{1,3} \cdot$ Marcel van Straten ${ }^{1}$
}

Received: 4 December 2019 / Revised: 21 January 2020 / Accepted: 4 February 2020 / Published online: 19 February 2020

(C) The Author(s) 2020

\begin{abstract}
Objective To assess the dose reduction potential of a calcium-aware reconstruction technique, which aims at tube voltageindependent computed tomography (CT) numbers for calcium.

Methods and materials A cardiothoracic phantom, mimicking three different patient sizes, was scanned with two calcium inserts (named D100 and CCI), containing calcifications varying in size and density. Tube voltage was varied both manually (range 70150 and $\mathrm{Sn} 100 \mathrm{kVp}$ ) and automatically. Tube current was automatically adapted to maintain reference image quality defined at $120 \mathrm{kVp}$. Data was reconstructed with the standard reconstruction technique (kernel Qr36) and the calcium-aware reconstruction technique (kernel Sa36). We assessed the radiation dose reduction potential (volumetric CT dose index values (CTDIvol)), noise (standard deviation (SD)), mean CT number (HU) of each calcification, and Agatston scores for varying $\mathrm{kVp}$. Results were compared with the reference acquired at $120 \mathrm{kVp}$ and reconstructed with Qr36.

Results Automatic selection of the optimal tube voltage resulted in a CTDIvol reduction of $22 \%$, $15 \%$, and $12 \%$ compared with the reference for the small, medium, and large phantom, respectively. CT numbers differed up to $64 \%$ for the standard reconstruction and $11 \%$ for the calcium-aware reconstruction. Similarly, Agatston scores deviated up to $40 \%$ and $8 \%$ for the standard and calcium-aware reconstruction technique, respectively.

Conclusion CT numbers remained consistent with comparable calcium scores when the calcium-aware image reconstruction technique was applied with varying tube voltage. Less consistency was observed in small calcifications with low density. Automatic reduction of tube voltage resulted in a dose reduction of up to $22 \%$.

Key Points

- The calcium-aware image reconstruction technique allows for consistent CT numbers when varying the tube voltage.

- Automatic reduction of tube voltage results in a reduced radiation exposure of up to $22 \%$.

- This study stresses the known limitations of the current Agatston score technique.
\end{abstract}

Keywords Computed x-ray tomography $\cdot$ Coronary artery disease $\cdot$ Radiation dosage $\cdot$ Diagnostic imaging

Electronic supplementary material The online version of this article (https://doi.org/10.1007/s00330-020-06709-9) contains supplementary material, which is available to authorized users.

Ronald Booij

r.booij@erasmusmc.nl

1 Department of Radiology \& Nuclear Medicine, Erasmus MC, P.O. Box 2240, 3000 CA Rotterdam, The Netherlands

2 Department of Radiology, University Medical Center Utrecht, Utrecht, The Netherlands

3 Department of Epidemiology, Erasmus MC, Rotterdam, The Netherlands

$\begin{array}{ll}\text { Abbreviations } \\ \text { ATCM } & \text { Automated tube current modulation } \\ \text { BAS } & \text { Background Agatston score } \\ \text { CACS } & \text { Coronary artery calcium scoring } \\ \text { CI } & \text { Confidence interval } \\ \text { CNR } & \text { Contrast to noise ratio } \\ \text { CT } & \text { Computed tomography } \\ \text { CTDI } & \text { Computed tomography dose index } \\ \text { DSCT } & \text { Dual source computed tomography } \\ \text { Dw } & \text { Water equivalent diameter } \\ \text { FBP } & \text { Filtered back projection } \\ \text { FoV } & \text { Field of view } \\ \text { HU } & \text { Hounsfield unit } \\ \text { ICC } & \text { Intraclass correlation coefficient } \\ \text { SD } & \text { Standard deviation }\end{array}$




\section{Introduction}

Ischemic heart diseases remain one of the leading causes of death worldwide $[1,2]$. Within the framework of individual risk prediction for these diseases, the assessment of coronary artery calcium has become increasingly important. Currently, the most common strategy for quantification of the coronary artery calcium score (CACS) is on computed tomography (CT) examinations using the Agatston method [3]. Despite the excellent prognostic value of this CT-based strategy, the Agatston scoring method has some limitations [4, 5]. Recent guidelines demand a fixed tube voltage of 120 peak kilo voltage $(\mathrm{kVp})$ in combination with filtered back projection (FBP) or iterative reconstruction with $100 \mathrm{kVp}$ acquisition after siteand literature-based validation [5, 6]. However, there is a main argument for the use of lower, or even patient-specific, tube voltages: the need to reduce radiation dose given the increase in the number of CT examinations [7].

Lowering tube voltage potentially reduces radiation dose in CACS at the cost of inconsistent scores because CT numbers, expressed in Hounsfield units (HU), are energy dependent. In this case, the standard calcium scoring threshold should be made tube voltage or patient-specific.

Recently, a calcium-aware reconstruction technique was introduced via the application of a new reconstruction kernel (Sa36f). The technique is also known by the name "Agatston score equivalent calcium scoring," "artificial $120 \mathrm{kV}$ equivalent CT images," or "artificial 120." Please refer to the vendor's whitepaper for a detailed explanation [8]. With this technique, CT numbers of calcium are scaled to match the CT numbers that would have been measured at $120 \mathrm{kVp}$, enabling the use of the standard $130 \mathrm{HU}$ threshold [9]. The technique might enable acquiring images at reduced radiation dose, while preserving the Agatston score and its risk assessment potential. In contrast to tube voltage-dependent threshold adjustments, the calcium-aware reconstruction technique seems an easy tool to implement clinically.

The purpose of our phantom study was to evaluate the calcium-aware reconstruction technique with regard to coronary calcium quantification for a wide range of tube voltages and calcifications varying in size and density and for different chest sizes. Moreover, the radiation dose reduction by automatic tube voltage selection was assessed for these cases.

\section{Materials and methods}

\section{Phantom}

An anthropomorphic (cardio) thoracic CT phantom (QRM Thorax, QRM GmbH) in combination with two different inserts was used for quantitative assessment of CACS both for the standard and the calcium-aware reconstruction technique.
One insert (D100, QRM GmbH) contained 100 calcifications of different diameters $(0.5$ to $2.0 \mathrm{~mm})$ and hydroxyapatite (HA) densities (90 to $540 \mathrm{mg} \mathrm{HA} / \mathrm{cm}^{3}$ ) [10]. The other insert was a cylindrical cardiac calcification insert (CCI, QRM $\mathrm{GmbH})$ with nine calcifications varying in size (1.0 to $5.0 \mathrm{~mm}$ ) and density (200 to $800 \mathrm{mg} \mathrm{HA} / \mathrm{cm}^{3}$ ). To simulate different chest sizes, the thorax phantom was scanned with and without fat-equivalent extension rings (QRM $\mathrm{GmbH})$ resulting in three different chest sizes: small $(300 \times$ $200 \mathrm{~mm})$, medium $(350 \times 250 \mathrm{~mm})$, and large $(400 \times$ $300 \mathrm{~mm}$ ). To ensure a realistic translation of the results from different phantom sizes to human chest sizes, the water equivalent diameter (Dw) was used. Dw reflects the x-ray attenuation of the patient and is therefore a preferred patient size metric [11]. Retrospective analysis of Dw's in 41 patient scans for CACS performed in our hospital showed that these diameters mostly matched with the Dw of the medium and large extension rings.

\section{Acquisition and reconstruction parameters}

Scans were performed on a dual source CT (DSCT) system (SOMATOM Force, Siemens Healthineers, Syngo CT VB10). A reference tube voltage of $120 \mathrm{kVp}$ in combination with automated tube current modulation (ATCM) CARE Dose4D was used for both inserts (Table 1). The calcium-aware reconstruction technique was assessed by acquiring data with varying tube voltages of $70-150 \mathrm{kVp}$, in steps of $10 \mathrm{kVp}$. Additionally, automatic tube voltage selection ("kVon") was set to keep the contrast to noise ratio for calcium constant when selecting the optimal tube voltage for radiation dose optimization. Finally, a scan was performed using a dedicated CACS Tin filtration protocol with an adaptation of the reference tube voltage to Sn100 in combination with ATCM CARE Dose4D (Table 1). All scans were repeated five times after manual repositioning (approximately $2 \mathrm{~mm}$ translation and 2 degrees rotation) of the phantom to assess positioning influence and interscan variation.

Images were reconstructed with the conventional calcium scoring reconstruction technique (kernel Qr36) and the dedicated calcium-aware reconstruction technique (kernel Sa36), both based on FBP. For the latter technique, calcium is identified in preliminary reconstructed images and a lookup table is used to correct the CT numbers of calcium in the finally reconstructed images [8]. The exact working of the algorithm is proprietary information of the vendor. The algorithm is fully integrated within the standard image reconstruction interface and can be activated by selecting the corresponding reconstruction kernel (Sa36). It does not need an additional workstation or increased reconstruction times. 
Table 1 Acquisition and reconstruction parameters

\begin{tabular}{lll}
\hline Scanner* & SOMATOM Force & SOMATOM Force-tin filtration \\
\hline Acquisition mode & Sequential & Sequential \\
Scan length (mm) & 100.5 & 100.5 \\
Reference tube voltage & 120 & Sn100 \\
Reference tube current product & 80 & 534 \\
Manual tube voltage settings & $70-150$ & Sn100 \\
CARE kV dose optimization slider** & 5 (bone/calcium) & 5 (bone/calcium) \\
Collimation (mm) & $32 \times 1.2$ & $32 \times 1.2$ \\
Rotation time (sec) & 0.25 & 0.25 \\
Image reconstruction (FBP) & Qr36 and Sa36 & Qr36 and Sa36 \\
Slice thickness (mm) & 3.0 & 3.0 \\
Increment (mm)*** & 1.5 & 1.5 \\
FoV (mm) & 180 & 180 \\
Reconstruction matrix & $512 \times 512$ & $512 \times 512$ \\
\hline
\end{tabular}

*Siemens Healthineers, Syngo CT VB10

**The dose optimization slider from the default calcium scoring protocol was retained

*** Increment of $1.5 \mathrm{~mm}$ is the standard for calcium scoring with Siemens equipment

\section{Image and dose analysis}

The volumetric CT dose index values (CTDIvol) in mGy were noted to assess potential radiation dose reduction. Consistency of CT numbers (mean and standard deviation (SD)) was determined in the central calcium insert (200HA) of the CCI insert. Noise SD was determined within a homogeneous region of the CCI insert. Agatston score, together with different image quality metrics, was computed using an in-house developed Python script (Python version 3.7) for the D100 and CCI insert. Resulting Agatston scores of the Python script were validated against the standard vendor-specific scoring software (Syngo.via, Siemens Healthineers) with the aid of $\mathrm{CCI}$ data and proven equal (maximum deviation $0.1 \%$ ).

This study addresses directly the CT number or CT value in Hounsfield units (HU) of calcifications. CT numbers are related to the linear x-ray attenuation coefficients and depend on the density, the effective atomic number, and x-ray tube voltage [12]. The attenuation coefficient of the phantom base material does not resemble the attenuation coefficient of human soft tissue equally well at all tube voltages. Allmendinger et al previously described a base material-specific correction, necessary for correct Agatston scores at varying tube voltages by adjustment of the standard $130 \mathrm{HU}$ threshold [8]. This correction was applied automatically in our study as well as for all reconstructions.

Image noise was compared with recommended noise targets (in HU) for calcium scoring CT scans defined for different chest sizes (small, medium, large chest width): $20 \mathrm{HU}$ for the small and medium chest width, and $23 \mathrm{HU}$ for the large chest width [13].

Additionally, an Agatston score was determined in a noncalcium region $(55 \times 55 \mathrm{~mm})$, therefore depending purely on noise. This score was called the background Agatston score (BAS). For acquisitions with a non-zero BAS, the Agatston scores of calcifications could be less reliable, as it was uncertain if a calcification was seen at a specific location, or just noise. These scores were noted.

Reference values for both inserts were the Agatston scores acquired with a tube voltage of $120 \mathrm{kVp}$ and reconstructed with the standard technique (Qr36). Each deviation in acquisition or reconstruction was compared against this reference

\section{Statistical analyses}

SPSS (version 25, IBM Corp) was used for statistical analysis. Normality of data was tested with the Shapiro-Wilk test. Wilcoxon signed-rank test was performed to evaluate statistically significant difference of the median Agatston scores. Intraclass correlation coefficients (ICC) with a $95 \%$ confidence interval (CI) and Bland-Altman plots of the Agatston scores between two different techniques were assessed. A $p$ value of $<0.05$ was considered statistically significant. Agatston scores are given as median values of the five measurements.

\section{Results}

\section{Radiation dose and noise values}

Reference dose levels at $120 \mathrm{kVp}$ for the small, medium, and large phantom size were $1.57,2.59$, and $3.84 \mathrm{mGy}$ respectively. For the scans with automatic tube voltage selection, tube voltage was reduced to $90 \mathrm{kVp}$ for the small and medium 
phantom size, while $100 \mathrm{kVp}$ was selected for the large phantom. In comparison with the corresponding reference, radiation dose levels decreased by $22 \%, 15 \%$, and $12 \%$ for the small, medium, and large phantom size, respectively.

Within the dedicated Tin CACS protocol, dose values were $55 \%$ lower for both small and medium phantom size and $60 \%$ for the large phantom size compared with the reference dose levels at $120 \mathrm{kVp}$.

Median noise values for the $120 \mathrm{kVp}$ and the images obtained with automatic tube voltage selection increased with increasing phantom diameter for both reconstruction techniques (Fig. 1). The noise level in all three phantom sizes was highest when using Tin filtration. Moreover, the recommended noise target for calcium scoring CT scans was exceeded for some tube voltages in the medium phantom and for all tube voltages in the large phantom size (Fig. 1). Despite the high number of noise limit exceeding scans, BAS values were zero for most reconstructions. A BAS $>0$ was found only for the large phantom in combination with a tube voltage of $70 \mathrm{kVp}$ or $\mathrm{Sn} 100$.

\section{CT number constancy}

Considering the large calcification with $200 \mathrm{mg} \mathrm{HA} / \mathrm{cm}^{3}$ in the CCI insert for all phantom sizes, CT numbers increased with decreasing tube voltage for the standard reconstruction technique, while these numbers remained virtually constant for the calcium-aware reconstruction technique (Table 2). Median HU (min HU-max HU) of the reference (120
$\mathrm{kVp}+\mathrm{Qr} 36)$ was $266 \mathrm{HU}(265 \mathrm{HU}-268 \mathrm{HU}), 257 \mathrm{HU}$ (257HU-258HU), and $247 \mathrm{HU}(246 \mathrm{HU}-248 \mathrm{HU})$ for the small, medium, and large phantom size, respectively. Compared with the reference, the deviation was up to $64 \%$ with the standard reconstruction technique and up to $11 \%$ with the calcium-aware reconstruction technique when varying the tube voltage (Table 2).

\section{Agatston score}

When varying the tube voltage, Agatston scores deviated up to $40 \%$ and $8 \%$ from the reference for the standard and calcium-aware reconstruction technique, respectively (Table 3). The overall spread in median Agatston scores for varying tube voltages decreased for the calcium-aware reconstruction technique for both the CCI and D100 insert (Fig. 2 and Fig. 3). Considering all phantom sizes, the Agatston scores in the CCI insert increased with $14 \%$ for the automated tube voltage selection and decreased with $14 \%$ within the tinfiltrated scans for the standard reconstruction technique (Fig. 2a). For the calcium-aware reconstruction technique, Agatston score deviations from the reference were much less: $3.6 \%$ at automated tube voltage selection and $2.4 \%$ with the tinfiltrated scans (Fig. 2b). For the D100 insert, we observed similar results; however, the deviations from the reference were larger than in the CCI insert, especially for the varying tube voltage in combination with the standard reconstruction technique (Fig. 3). Representative images of the D100 insert for the standard reconstruction technique with $120 \mathrm{kVp}$ and
Fig. 1 Box-and-whisker plots of the noise measurements of the homogeneous central slice of the $\mathrm{CCI}$ insert. Recommended noise targets (in $\mathrm{HU}$ ) for calcium scoring CT scans defined for different chest sizes were applied to the images as dotted lines: 20 HU for the small and medium chest width, and $23 \mathrm{HU}$ for the large chest width. The automatic tube voltage selection is illustrated by "kVon"

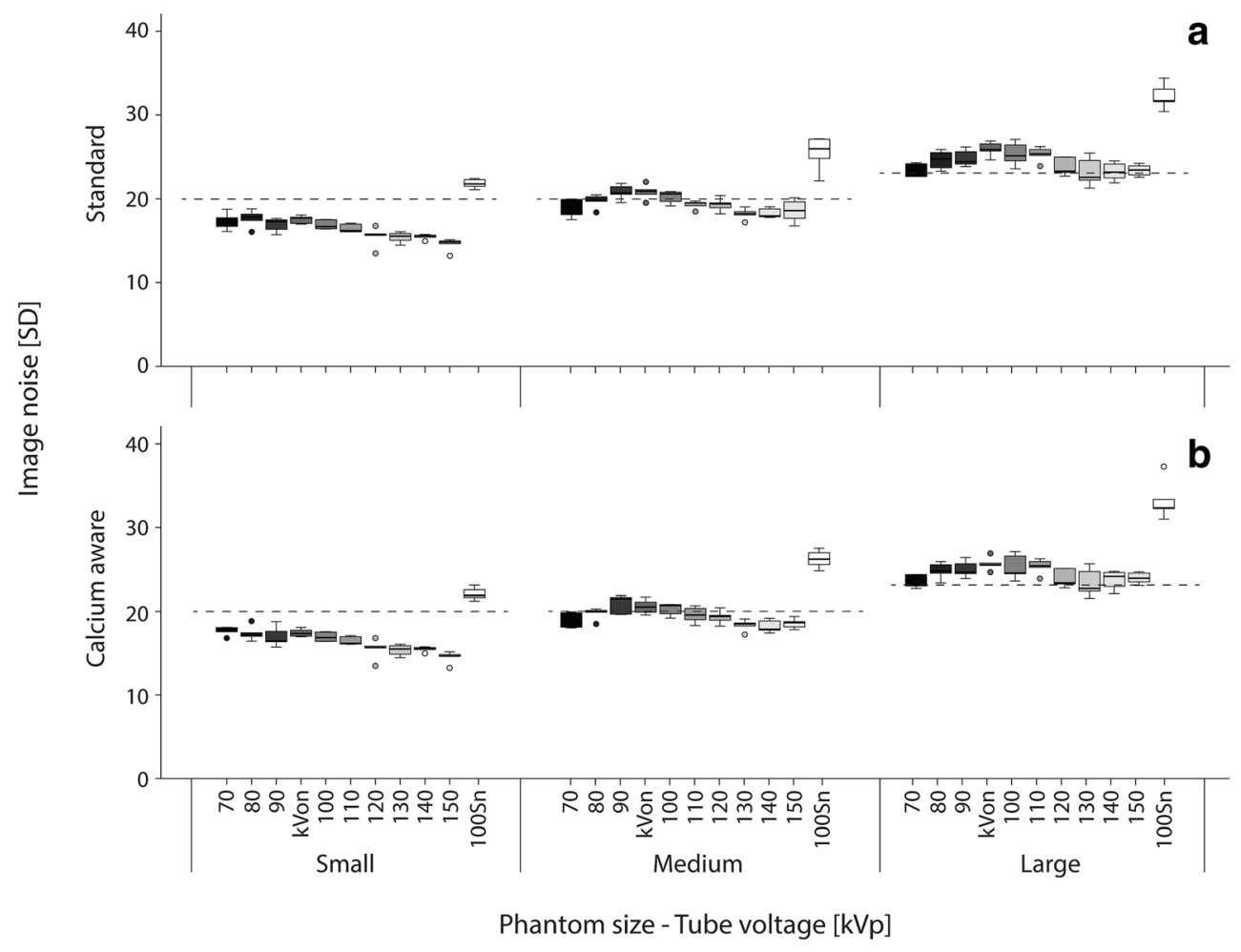




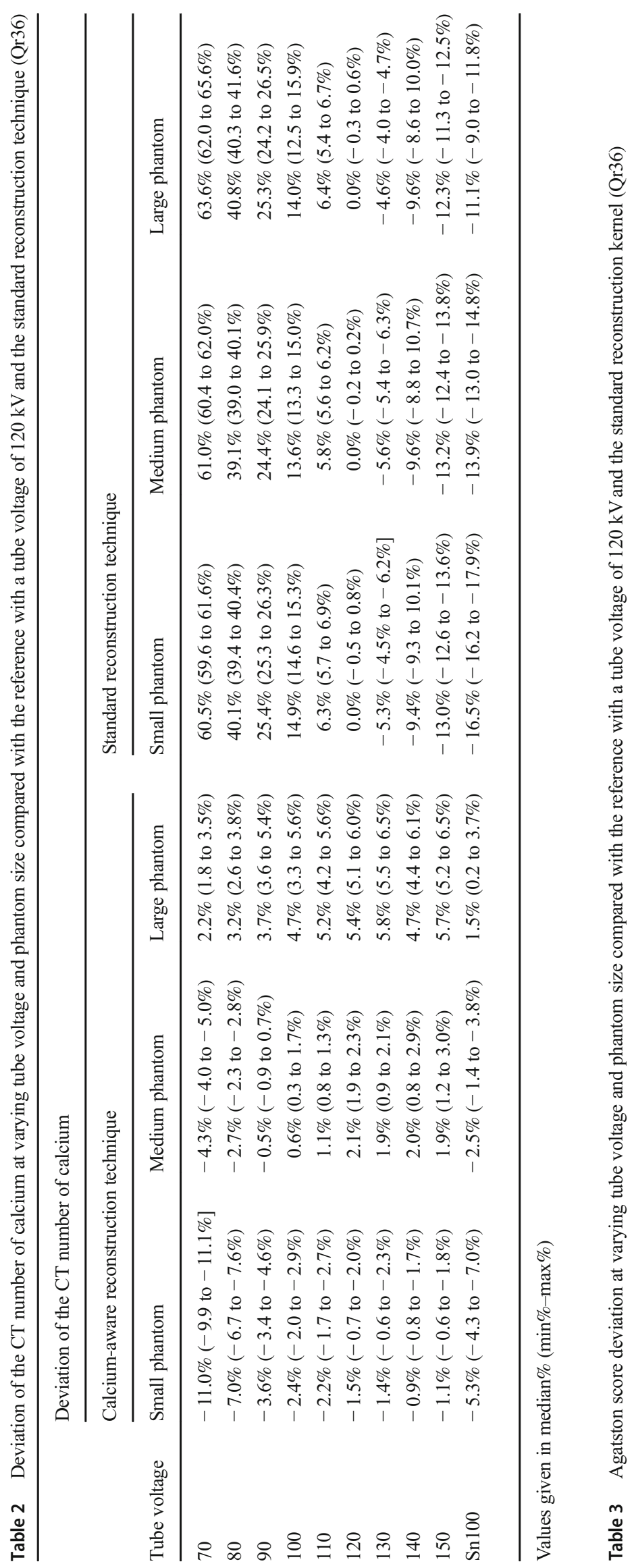

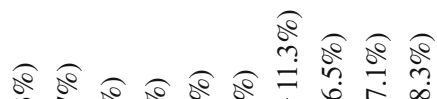

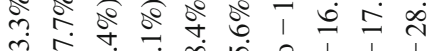

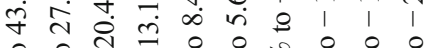
\% 8 ก 불

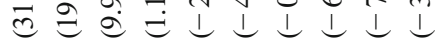

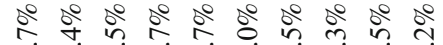

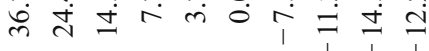

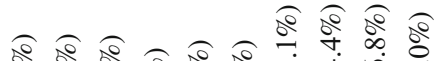

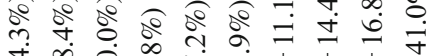

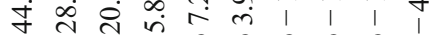

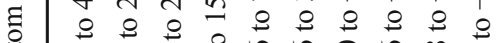

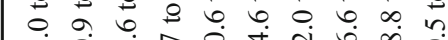

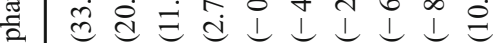

药 $\sum^{e}$

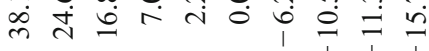

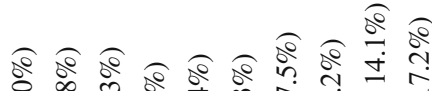

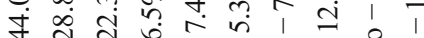

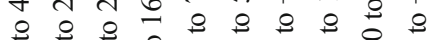
b 0 a 9 m $t$ n m 0

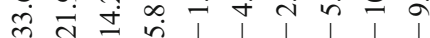

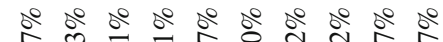
ले ठ઼

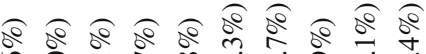

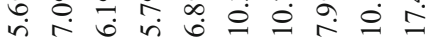

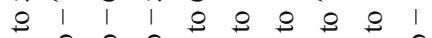

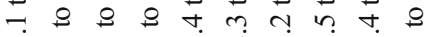

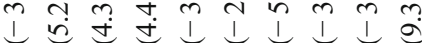

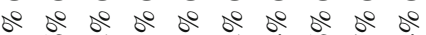

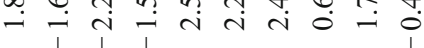

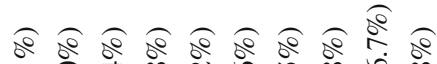

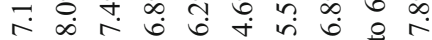

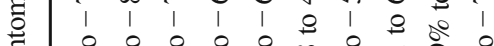

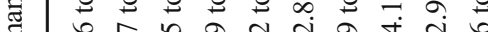

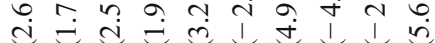

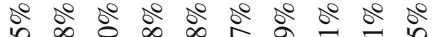
$\begin{array}{llllllllll}1 & 1 & 1 & 1 & 1 & 0 & 1 & 1 & 0 & 1\end{array}$

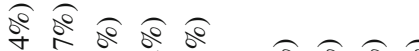

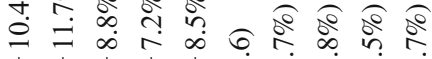

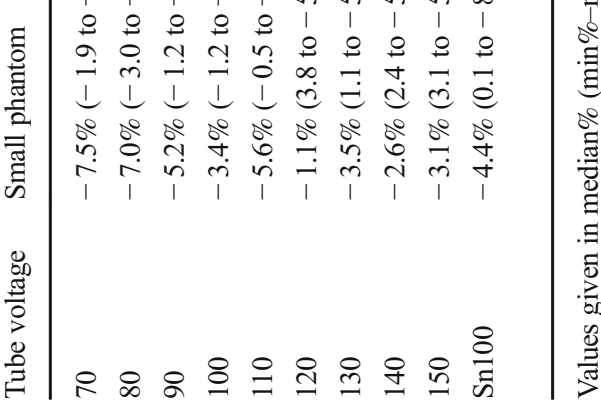


Fig. 2 a Box-and whisker plots of the Agatston score within the CCI insert with the standard reconstruction technique. b Box-andwhisker plots of the Agatston score within the CCI insert with the calcium-aware reconstruction technique. Scores are given per phantom size-tube voltage combination. The automatic tube voltage selection is illustrated by "kVon"

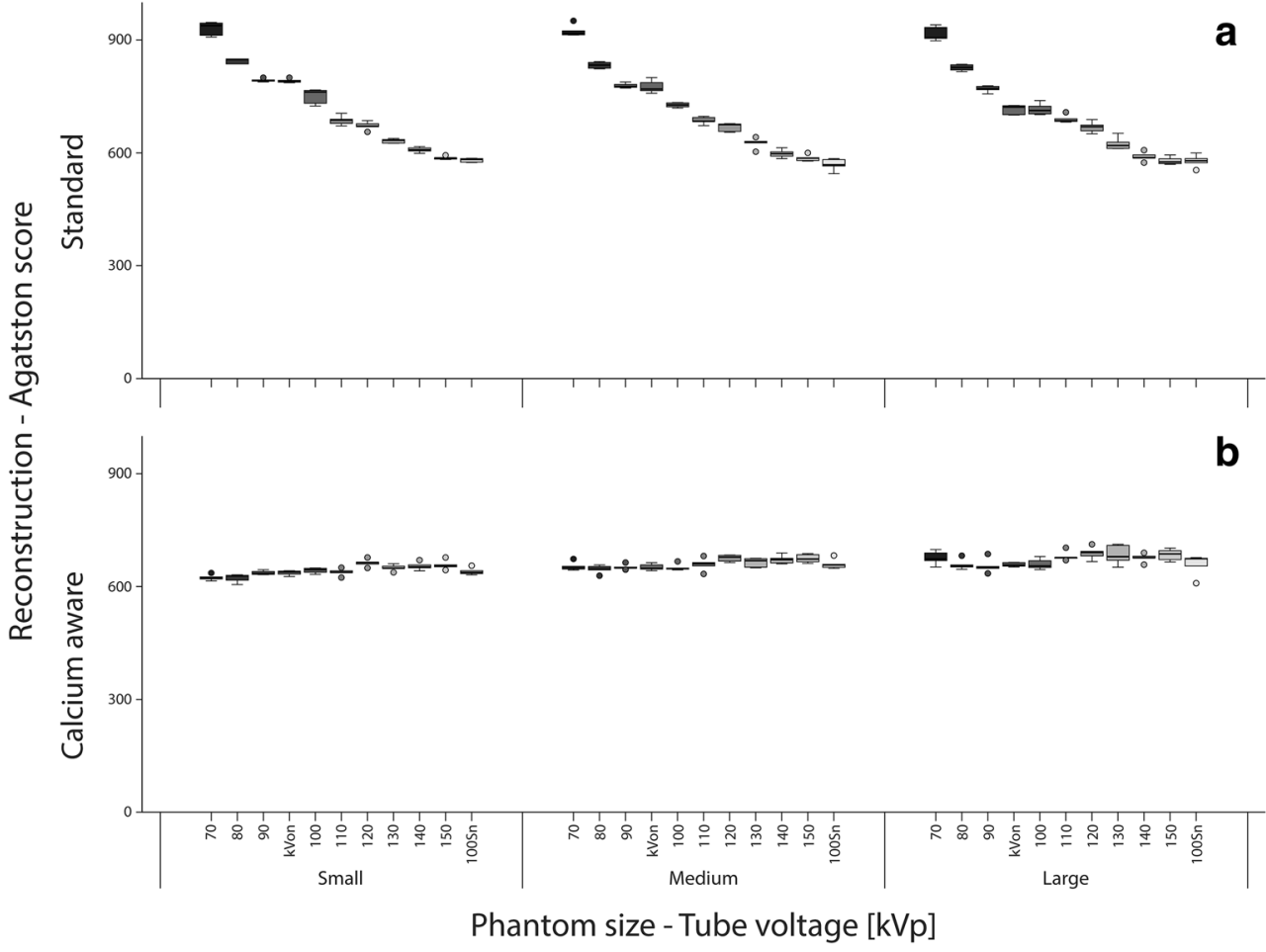

the calcium-aware reconstruction technique at reduced tube voltage for all three phantom sizes are shown in Fig. 4. This figure shows calcifications with an Agatston score of zero for the reference, while the calcium-aware reconstruction technique Agatston scores are non-zero.
There was a very high ICC (0.991) and 95\% CI for the automated tube voltage selection with the standard reconstruction technique compared with the reference when considering all calcifications (Fig. 5a). When considering only the low Agatston scores, both the ICC and 95\% CI decreased (Fig.
Fig. 3 a Box-and-whisker plots of the Agatston score within the D100 insert with the standard reconstruction technique. b Boxand whisker-plots of the Agatston score within the D100 insert with the calcium-aware reconstruction technique. Scores are given per phantom size-tube voltage combination. The automatic tube voltage selection is illustrated by "kVon"

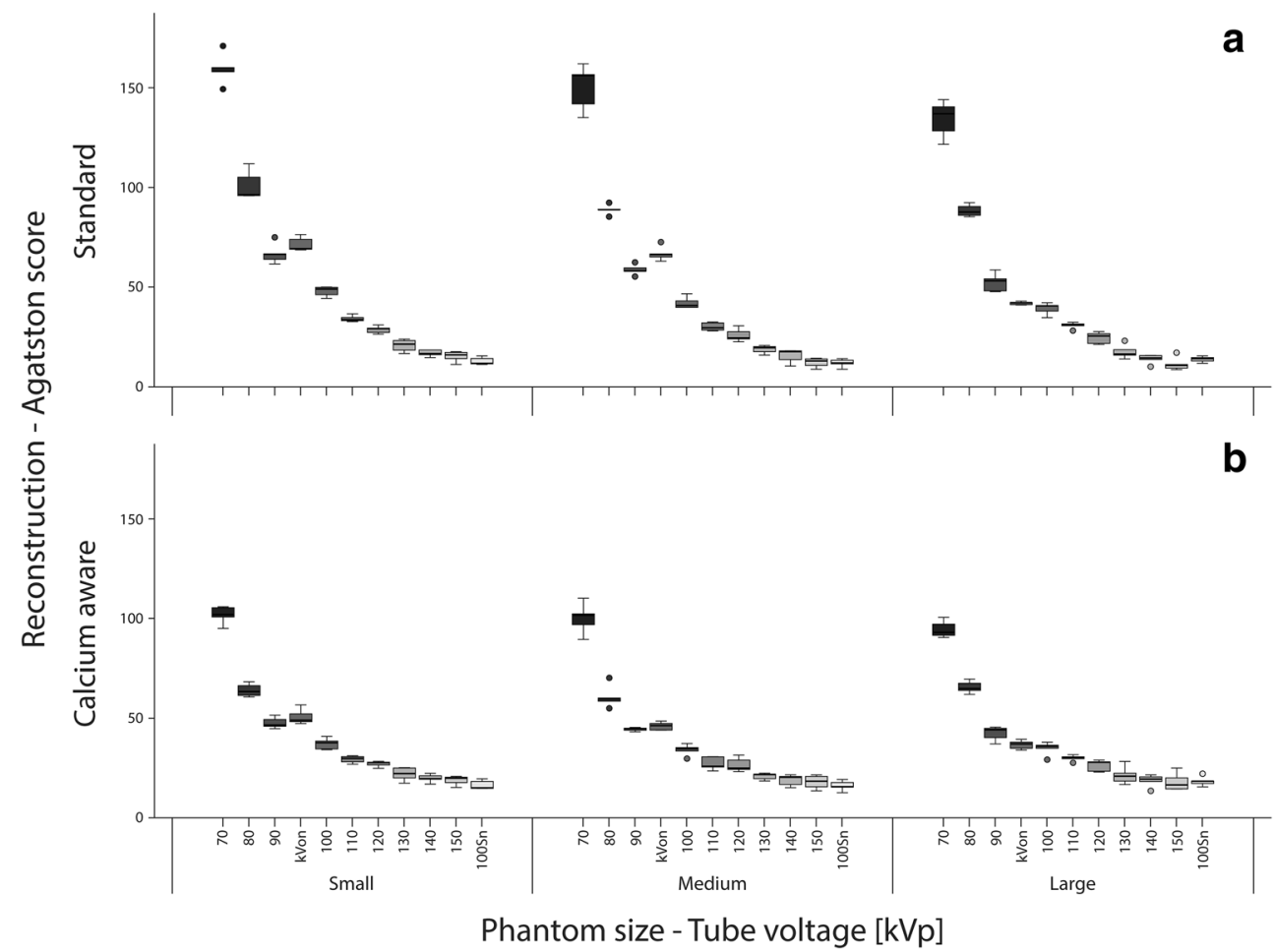


Fig. 4 Visualization of calcifications in the D100 insert with all voxels with a CT number above the threshold colored red. From left to right, the phantom size increases. The upper row images were reconstructed with the standard reconstruction technique with a tube voltage of $120 \mathrm{kVp}$. Lower row images were reconstructed with the calciumaware reconstruction technique and automated tube voltage selection $(90 \mathrm{kVp}$ for the small and medium size phantom and 100 $\mathrm{kVp}$ for the large size phantom)

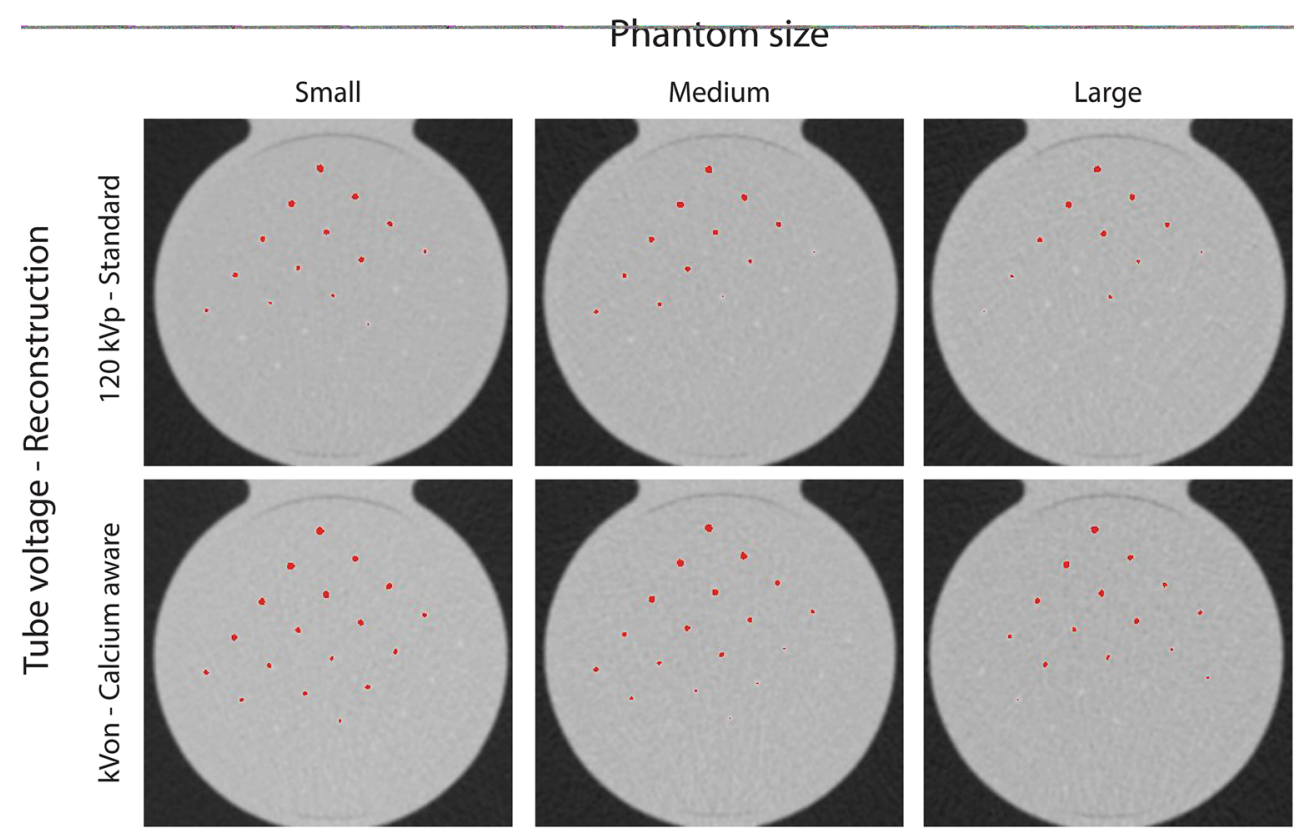

5b). There was a very high ICC (0.998) and 95\% CI for the automated tube voltage selection and the calcium-aware reconstruction technique compared with the reference (Fig. 5c). When considering only the low Agatston scores, both the ICC and $95 \%$ CI decreased (Fig. 5d). However, this decrease was less than observed within the standard reconstruction technique. A Bland-Altman analysis of the data is shown in Fig. 6. The Bland-Altman plots demonstrate the agreement between the two reconstruction kernels. The negative mean difference within Fig. 6 a, b, and d demonstrates that, regardless of reconstruction technique, Agatston scores are higher for automatic tube voltage selection in comparison with 120 $\mathrm{kVp}$. The opposite applies for the calcium-aware reconstruction technique and automatic tube voltage selection (Fig. 6c).

For the CCI insert, increasing the phantom diameter from small to large demonstrated no statistically significant
Fig. 5 The ICC of the Agatston score for the small, medium, and large phantom for. a The standard reconstruction technique with automatic tube voltage selection compared with the standard reconstruction with $120 \mathrm{kVp}$. b Detail of the graph in a representing the low density and small calcifications. c The calcium-aware reconstruction technique with automatic tube voltage selection and the standard reconstruction with $120 \mathrm{kVp}$. d Detail of the graph in $\mathbf{c}$ representing the low density and small calcifications

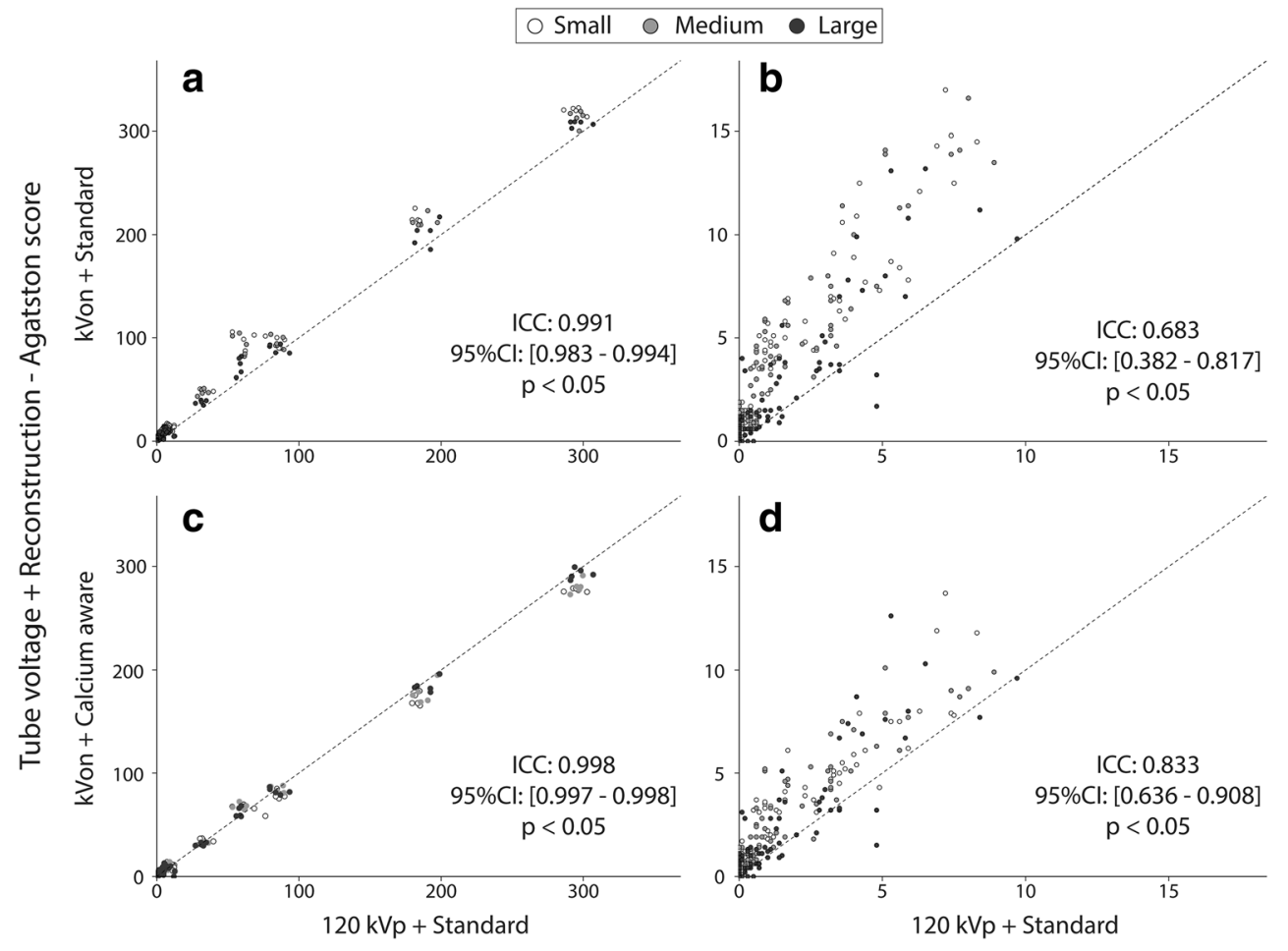

Tube voltage + Reconstruction - Agatston score 
Fig. 6 Bland-Altman Plots with mean difference and 95\% limits of agreement for the small, medium, and large phantom with the CCI and/or D100 insert. All plots show an Agatston score comparison between the reference at $120 \mathrm{kVp}$ (with standard reconstruction technique) and scans with automatic tube voltage selection (with standard reconstruction technique $(\mathbf{a}, \mathbf{b})$ and with calcium-aware reconstruction technique $(\mathbf{c}, \mathbf{d}))$

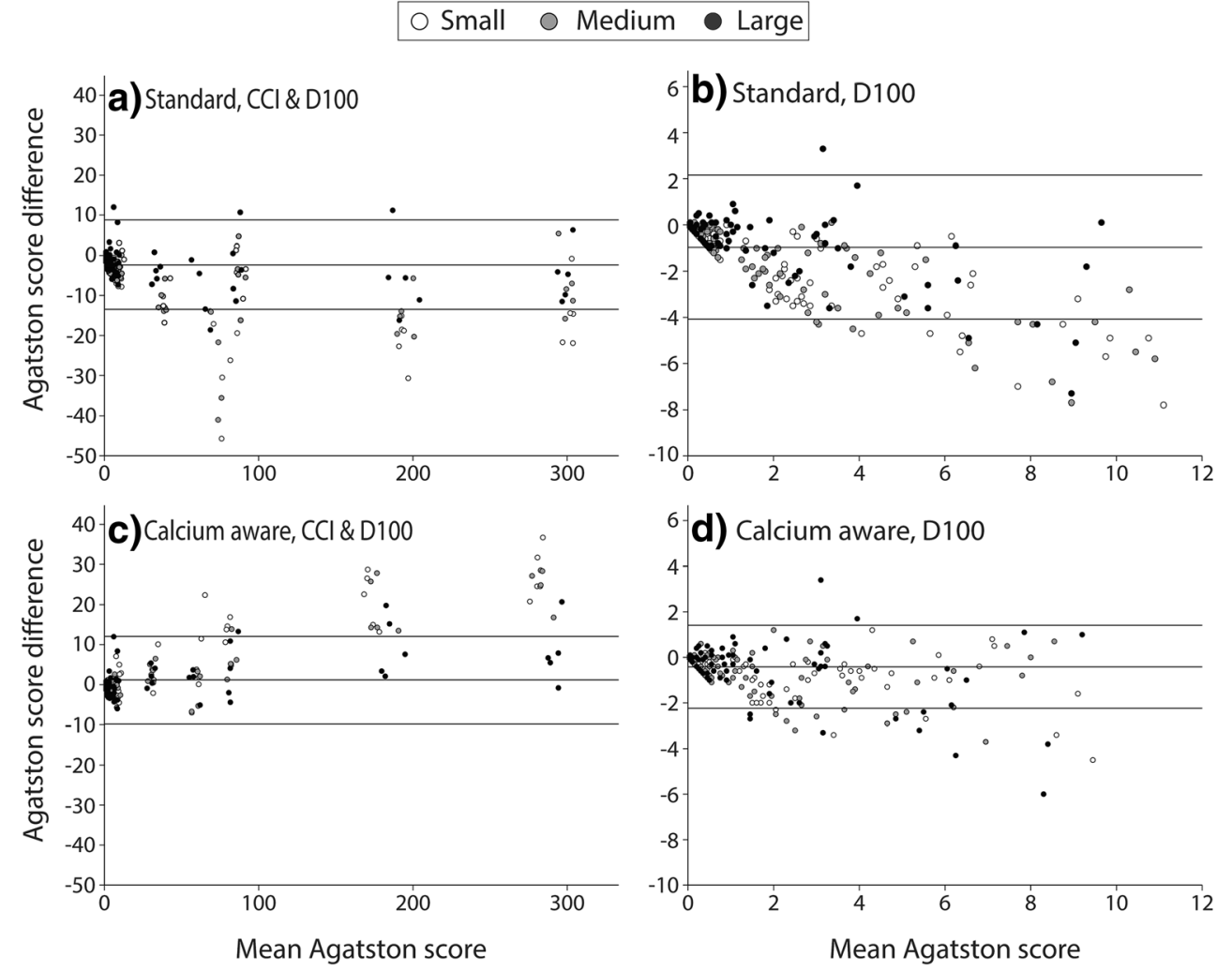

decrease $(p=0.5)$ of the median (range) Agatston scores from $671(656.2$ - 686.5) to $669.9(651.1$ - 689.4) for the reference $(120 \mathrm{kVp}$ and Qr36). A statistically significant increase $(p<0.05)$ in Agatston score from 639 (626.9-642.4) to 657.4 (652-664.5) was observed for the calcium-aware technique with automated tube voltage selection. For the D100 insert and increasing phantom size from small to large, there was a statistically significant decrease $(p<0.05)$ in Agatston score from $29.3(26.5-31.2)$ to $25.6(21.4-27.8)$ for the reference $(p<0.05)$, and a statistically significant decrease $(p<0.05)$ in Agatston score from 49.0 (47.4-56.7) to 37.1 $(34.0$-39.4) for the calcium-aware reconstruction technique with automatic tube voltage selection.

\section{Discussion}

Our results demonstrate that CACS with a calcium-aware image reconstruction technique allows for consistent $\mathrm{CT}$ numbers when varying the tube voltage and allows for reduced radiation exposure with automatic reduction of tube voltage.

The Agatston scores with the calcium-aware reconstruction technique deviated up to $8 \%$ for the calcifications of the CCI insert across 70 to $150 \mathrm{kVp}$ and $\mathrm{Sn} 100$, whereas the Agatston score with the standard reconstruction deviated much more with up to $40 \%$. The latter might be explained by the increase of the photo-electric effect for calcium when scanning with low tube voltage settings. In contrast to the CCI insert, Agatston scores were not stable for the calcifications of the D100 insert when varying the tube voltage. For the calciumaware reconstruction technique, this might be explained by a sub-optimal identification of the voxels containing calcifications of small diameter and low density and subsequently a sub-optimal correction of the CT numbers.

As seen in Fig. 4, there were additional calcifications detected when lowering tube voltage. Thus, it might be possible that a patient with a zero Agatston score at $120 \mathrm{kVp}$ might have a non-zero Agatston score at a lower tube voltage, despite the application of the calcium-aware reconstruction technique. This might influence the work-up of patients suspected for coronary artery disease. However, the increase of Agatston score in the D100 insert, as demonstrated in Fig. 4, is due to true calcified lesions. Instead of improving the calcium-aware reconstruction technique presented in this study to better resemble the Agatston scores at $120 \mathrm{kV}$, we prefer to reinvent calcium imaging and think it is time to let go the conventional scoring method $[14,15]$. For example, Groen et al described a correction applied to the $130 \mathrm{HU}$ calcium scoring threshold for the increased CT numbers of calcium when varying tube voltage and applying the standard reconstruction technique [16].

Our study demonstrated a decrease in Agatston score with increasing phantom size, as previously described for the standard reconstruction technique and the D100 insert [17]. 
However, our study used both the CCI and the D100 insert and in addition the calcium-aware reconstruction technique. We observed an increase of the Agatston score for the CCI insert when using the calcium-aware reconstruction technique. The increase in Agatston score might be explained by the sub-optimal identification of the voxels containing small and low-density calcifications, while noise increased.

Calcium CT numbers were constant for the calcium-aware reconstruction technique with automated tube voltage selection, irrespective of phantom size. However, Agatston scores varied more than the reference for different patient sizes. The reason for this is twofold. First, the constancy of CT numbers is calculated as the mean of a large ROI enclosing the calibration rod of the CCI phantom, while Agatston scores are calculated for the smaller nine calcifications. Second, despite the use of clinical scan protocols, higher noise levels were shown especially for the lower tube voltages and the automated tube voltage selection (Fig. 1). Our computation of the Agatston score was validated to the standard vendor-specific software, calculating every single voxel above a threshold of $130 \mathrm{HU}$ for CACS. With higher noise levels, Agatston scores also increase.

Technological developments like tin filtration and automated tube voltage selection allow for a substantial dose reduction. For example, a $100 \mathrm{kVp}$ with tin filtration CACS protocol demonstrated similar Agatston scores as the reference protocol with $120 \mathrm{kVp}$ despite using the standard reconstruction technique [18]. Larger deviations are expected for tube voltages like 70 and $80 \mathrm{kVp}$ (Table 2). A great advantage of the currently considered calcium-aware reconstruction technique is that CACS can be obtained more accurately from any acquisition, regardless of applied tube voltage and filtration. This allows for CACS to be considered within cancer screening protocols. The use of a CACS with the aid of tin filtration combined with an early prototype of a calcium-aware reconstruction technique was described in a patient study and considered to be potentially feasible for calcium scoring [19]. However, in this study and our study, an increased image noise for the tin-filtrated scans was observed. The noise levels were above the recommended noise levels by the SCCT in all three phantom sizes, especially for the large phantom size. Possible solutions for sub-optimal identification of calcification when applying tin filtration with increased noise levels are proposed, e.g., a HU threshold correction for CACS [20] or investigation to apply iterative reconstructions. Within our study, we observed BAS of $>0$ for the tin-filtrated vendor-recommended scans in the large phantom size. Therefore, caution must be taken when applying the tin-filtrated scans in clinical routine, especially when CACS is obtained for calcification of small diameter and low density, as the calcium-aware reconstruction technique is also not able to correct these.

The recommended noise levels were not only exceeded for the tin-filtrated scanning protocols, but also for all tube voltage settings within the large phantom diameter, despite the use of the vendor-recommended scanning protocols. This warrants further investigation for adjusting the reference tube current value or the adaptation strength of the CARE Dose4D dose curve to achieve the recommended noise target level [13]. However, it seems that the recommended noise target limit comes with a very safe margin. After all, the BAS was zero for all reconstructions in the small- and medium-sized phantoms and for the calcium-aware reconstruction technique with automated tube voltage selection in all phantoms.

There are limitations in this study that need to be considered. This study was phantom-based and despite the effort to represent clinical routine, patient studies are necessary to validate our findings. CTDIvol is an indicator of the CT scanner radiation output. The dose received by a patient depends on this CTDIvol and the individual patient size. It is recommended to use the size-specific dose estimates (SSDE) to reflect estimated doses for the individual patient [21]. Furthermore, it might be of interest to use a non-stationary phantom model instead of a stationary one. This makes it feasible to assess whether or not heart rate variability will influence Agatston scores when using the calcium-aware reconstruction technique.

\section{Conclusion}

In general, CT numbers remained consistent with comparable calcium scores when the calcium-aware image reconstruction technique was applied with varying tube voltage. Less consistency was observed in small calcifications with low density. Automatic reduction of tube voltage resulted in a dose reduction of up to $22 \%$.

Acknowledgments We would like to thank Maarten Kremer and Marcel Dijkshoorn (Erasmus MC, Rotterdam) for their support during scanning and Marcel Greuter, PhD (University medical center Groningen, Groningen), for lending the D100 insert.

Funding information The authors state that this work has not received any funding.

\section{Compliance with ethical standards}

Guarantor The scientific guarantor of this publication is Marcel van Straten.

Conflict of interest The authors of this manuscript declare relationships with the following companies:

R. Booij: Research collaboration, Siemens Healthineers

N. van der Werf: None

R.P.J. Budde: None

D. Bos: None

M. van Straten: Research collaboration, Siemens Healthineers

Our department has a Master Research Agreement with Siemens Healthineers. 
Statistics and biometry One of the authors has significant statistical expertise.

Informed consent Written informed consent was not required for this study because it was phantom based.

Ethical approval Institutional Review Board approval was not required because it was phantom based.

\section{Methodology \\ - Experimental \\ - Performed at one institution}

Open Access This article is licensed under a Creative Commons Attribution 4.0 International License, which permits use, sharing, adaptation, distribution and reproduction in any medium or format, as long as you give appropriate credit to the original author(s) and the source, provide a link to the Creative Commons licence, and indicate if changes were made. The images or other third party material in this article are included in the article's Creative Commons licence, unless indicated otherwise in a credit line to the material. If material is not included in the article's Creative Commons licence and your intended use is not permitted by statutory regulation or exceeds the permitted use, you will need to obtain permission directly from the copyright holder. To view a copy of this licence, visit http://creativecommons.org/licenses/by/4.0/.

\section{References}

1. Eurostat (2018) Statistics causes of death in the European Union (EU) 2005 - 2015. Available via https://ec.europa.eu/eurostat/ statistics-explained/index.php/Causes of death statistics

2. Greenland P, Alpert JS, Beller GA et al (2010) 2010 ACCF/AHA guideline for assessment of cardiovascular risk in asymptomatic adults: a report of the American College of Cardiology Foundation/American Heart Association Task Force on Practice Guidelines. J Am Coll Cardiol 56:e50-e103

3. Elias-Smale SE, Proenca RV, Koller MT et al (2010) Coronary calcium score improves classification of coronary heart disease risk in the elderly: the Rotterdam study. J Am Coll Cardiol 56:14071414

4. Willemink MJ, van der Werf NR, Nieman K, Greuter MJW, Koweek LM, Fleischmann D (2018) Coronary artery calcium: a technical argument for a new scoring method. J Cardiovasc Comput Tomogr. https://doi.org/10.1016/j.jcct.2018.10.014

5. Hecht HS, Cronin P, Blaha MJ et al (2017) 2016 SCCT/STR guidelines for coronary artery calcium scoring of noncontrast noncardiac chest CT scans: a report of the Society of Cardiovascular Computed Tomography and Society of Thoracic Radiology. J Cardiovasc Comput Tomogr 11:74-84

6. Hecht H, Blaha MJ, Berman DS et al (2017) Clinical indications for coronary artery calcium scoring in asymptomatic patients: expert consensus statement from the Society of Cardiovascular Computed Tomography. J Cardiovasc Comput Tomogr 11:157-168
7. Bijwaard H, Pruppers M, de Waard-Schalkx I (2014) The influence of population aging and size on the number of $\mathrm{CT}$ examinations in the Netherlands. Health Phys 107:80-82

8. Allmendinger T, Hamann A (2018) Agatston calcium quantification with arbitrary tube voltage - white paper. Available via https:// www.siemens-healthineers.com/computed-tomography/clinicalimaging-solutions/cardiovascular-imaging\#CLINICAL USE

9. Agatston AS, Janowitz WR, Hildner FJ, Zusmer NR, Viamonte M Jr, Detrano R (1990) Quantification of coronary artery calcium using ultrafast computed tomography. J Am Coll Cardiol 15:827832

10. Groen JM, Kofoed KF, Zacho M, Vliegenthart R, Willems TP, Greuter MJ (2013) Calcium score of small coronary calcifications on multidetector computed tomography: results from a static phantom study. Eur J Radiol 82:e58-e63

11. McCollough C, Bakalyar DM, Bostani M et al (2014) Use of water equivalent diameter for calculating patient size and size-specific dose estimates (SSDE) in CT: the report of AAPM task group 220. AAPM Rep 2014:6-23

12. Lamba R, McGahan JP, Corwin MT et al (2014) CT Hounsfield numbers of soft tissues on unenhanced abdominal CT scans: variability between two different manufacturers' MDCT scanners. AJR Am J Roentgenol 203:1013-1020

13. Voros S, Rivera JJ, Berman DS et al (2011) Guideline for minimizing radiation exposure during acquisition of coronary artery calcium scans with the use of multidetector computed tomography: a report by the Society for Atherosclerosis Imaging and Prevention Tomographic Imaging and Prevention Councils in collaboration with the Society of Cardiovascular Computed Tomography. J Cardiovasc Comput Tomogr 5:75-83

14. Blaha MJ, Mortensen MB, Kianoush S, Tota-Maharaj R, CainzosAchirica M (2017) Coronary artery calcium scoring: is it time for a change in methodology? JACC Cardiovase Imaging 10:923-937

15. Nieman K (2015) Evolve or perish for coronary calcium imaging. Eur Heart J Cardiovasc Imaging 16:354-355

16. Groen JM, Dijkstra H, Greuter MJ, Oudkerk M (2009) Threshold adjusted calcium scoring using CT is less susceptible to cardiac motion and more accurate. Med Phys 36:438-446

17. Willemink MJ, Abramiuc B, den Harder AM et al (2015) Coronary calcium scores are systematically underestimated at a large chest size: a multivendor phantom study. J Cardiovasc Comput Tomogr 9:415-421

18. Apfaltrer G, Albrecht MH, Schoepf UJ et al (2018) High-pitch lowvoltage CT coronary artery calcium scoring with tin filtration: accuracy and radiation dose reduction. Eur Radiol 28:3097-3104

19. Tesche C, De Cecco CN, Schoepf UJ et al (2017) CT coronary calcium scoring with tin filtration using iterative beam-hardening calcium correction reconstruction. Eur J Radiol 91:29-34

20. Vonder M, Pelgrim GJ, Huijsse SE et al (2017) Feasibility of spectral shaping for detection and quantification of coronary calcifications in ultra-low dose CT. Eur Radiol 27:2047-2054

21. Brink JA, Morin RL (2012) Size-specific dose estimation for CT: how should it be used and what does it mean? Radiology 265:666668

Publisher's note Springer Nature remains neutral with regard to jurisdictional claims in published maps and institutional affiliations. 